\title{
Scattering of anti-plane waves by scalene trapezoidal boundary with embedded cavity in anisotropic material based on mapping space
}

\author{
Yingchao Sun ${ }^{1}$, Zailin Yang ${ }^{1}$, Yuliang $\mathrm{Li}^{2}$, and Haibin Lin ${ }^{2}$ \\ ${ }^{1}$ Harbin Engineering University \\ ${ }^{2}$ AVIC-HONGDU
}

December 3, 2021

\begin{abstract}
Both surface motion and hole stress concentration have always been concerned in anisotropic medium. In this paper, a theoretical approach is used to study the scattering problem of circular holes under a scalene trapezoid on the surface. The mapping function that anisotropic medium to homogeneous medium is established, and the relationship between the free boundary of anisotropic medium and the mapping of homogeneous medium boundary is proved. In the space of homogeneous medium mapping, the wave displacement function is obtained by solving the equation of motion that meets the zero-stress boundary conditions by separating the variable method and the symmetric method. Based on the complex function, multi-polar coordinate method and region-matching technique, algebraic equations are established at auxiliary boundary and free boundary conditions in complex domain. Then according to sample statistics, least square method is used instead of the Fourier expansion method to solve the undetermined coefficient of the algebraic equations by discrete boundary. Numerical results shows that the continuity of the auxiliary boundary and the accuracy of the zero-stress boundary are nice, and the displacement of the free surface and the stress of the circular hole are related to the parameters of material medium, the position of the circular hole, the direction of the incident wave and the frequency content of the excitation. Finally the process of the wave propagation and scattering around trapezoid and shallow circle are shown in time domain through the inverse fourier transform.
\end{abstract}

\section{Hosted file}

main document1.pdf available at https://authorea.com/users/449346/articles/547890-scatteringof-anti-plane-waves-by-scalene-trapezoidal-boundary-with-embedded-cavity-in-anisotropicmaterial-based-on-mapping-space 